\title{
Venous Ectasia of Retromandibular and Common Facial Veins: A Rare Clinical Entity
}

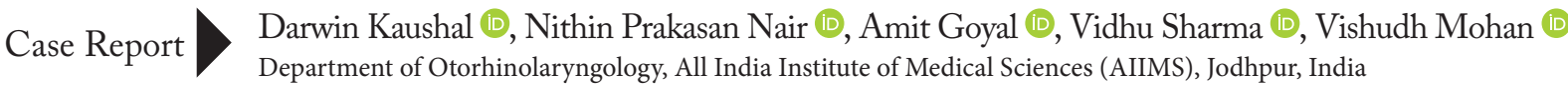

\begin{abstract}
Venous ectasias are benign conditions of the neck, in which focal dilatations of veins occur. Internal jugular, external jugular or superficial veins are usually the affected ones in the neck. They are often ignored or misdiagnosed. Here we are reporting a patient with venous ectasia of the retromandibular vein and the common facial vein. A 25-year-old male presented to our out-patient department with an intermittent swelling over the right side of the neck that he had for one year. The swelling was more prominent on lying down and on Valsalva maneuver. Radiologic imaging was suggestive of venous ectasia of the retromandibu-
\end{abstract}

\begin{abstract}
ORCID iDs of the authors: D.K. 0000-0001-7719-6409; N.P.N. 0000-0001-7342-9682; A.G. 0000-0002-4339-7541; V.S. 0000-0002-3547-2329; V.M. 0000-0002-0494-0812
\end{abstract}

Cite this article as: Kaushal D, Nair NP, Goyal A, Sharma V, Mohan V. Venous Ectasia of Retromandibular and Common Facial Veins: A Rare Clinical Entity. Turk Arch Otorhinolaryngol 2020; 58(4): $282-5$.

\section{Corresponding Author:}

Darwin Kaushal; drdarwin.aiims@gmail.com

Received Date: 15.05 .2020

Accepted Date: 17.10.2020

Content of this journal is licensed under a Creative Commons Attribution 4.0 International License. Available online at www.turkarchotolaryngol.net

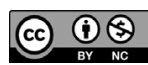

DOl: $10.5152 / \operatorname{ta} 0.2020 .5506$

\section{Introduction}

Venous ectasia in the neck is a rare and benign condition. It is the dilatation or aneurysm of the venous system of the neck that may arise from the internal jugular, external jugular or superficial veins. Patients with venous ectasia present with intermittent neck swelling and rarely with pain. The etiology is mainly trauma or thoracic outlet obstruction or tumor (1). There are very few cases of venous ectasia of the common facial vein and the retromandibular vein in the literature. Here, we report a young male who presented with intermittent neck swelling which eventually turned out to be a venous ectasia of the retromandibular and the common facial veins.

\section{Case Presentation}

A twenty-five-year-old gentleman presented to our outpatient department with complaints of painless swelling over the right-side neck for one year. There was a history of increased size of the swelling while lying down and coughing as seen in Figure 1. The swelling used to disappear while sitting or in erect position. There was no history of trauma. The patient is working for the armed forces; hence, we could not rule out the probability of non-trivial injury. There was no history of fever. There was no history of dyspnea, dysphagia or change in voice. lar vein and the common facial vein. Surgical excision was done for aesthetic reasons and in fear of thrombosis. Intraoperatively, we noticed that it was arising from the retromandibular and the common facial veins. Venous ectasias of superficial veins are rare. We can consider these patients for surgical excision in view of the risk of thrombosis, thromboembolic events, rupture, and aesthetic reasons.

Keywords: Ectasia, aneurysm, jugular vein, vein thrombosis, arteriovenous malformation, Valsalva maneuver
There was no visible swelling on inspection at presentation. A diffuse swelling was more prominent over the right side neck when the patient was in right lateral position. On palpation, a diffuse, non-tender smooth-surfaced and soft swelling of $5 \times 4 \mathrm{~cm}$ was palpable below the right side angle of the mandible, extending up to a middle third of the sternocleidomastoid muscle. The swelling was compressible and non-pulsatile. It was getting prominent on digital compression over the right side of the neck and during Valsalva maneuver. There was cough impulse over swelling. No other mass was palpable.

Contrast-enhanced computed tomography of the neck revealed ectatic dilatation of the venous system in the region of the retromandibular vein, near its confluence with the facial vein, forming a common facial vein as seen in Figures 2 and 3. Color doppler ultrasonography showed swirling flow with color filling and communication with the right side retromandibular vein and the common facial vein. Blood investigations including lipid profile, coagulation profile, D-dimer test and fibrinogen degradation product were done and all found normal. We considered a differential diagnosis of venous ectasia, lymphangioma and branchial cyst. Sclerotherapy was considered for this 


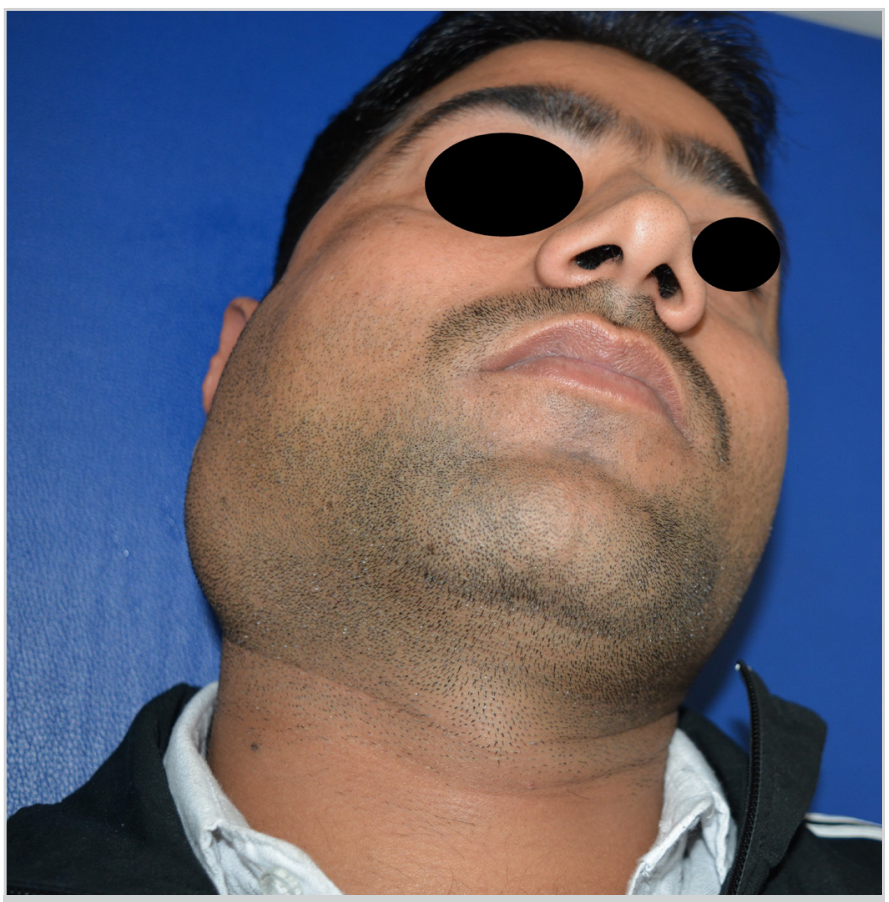

Figure 1. Prominent swelling on supine position

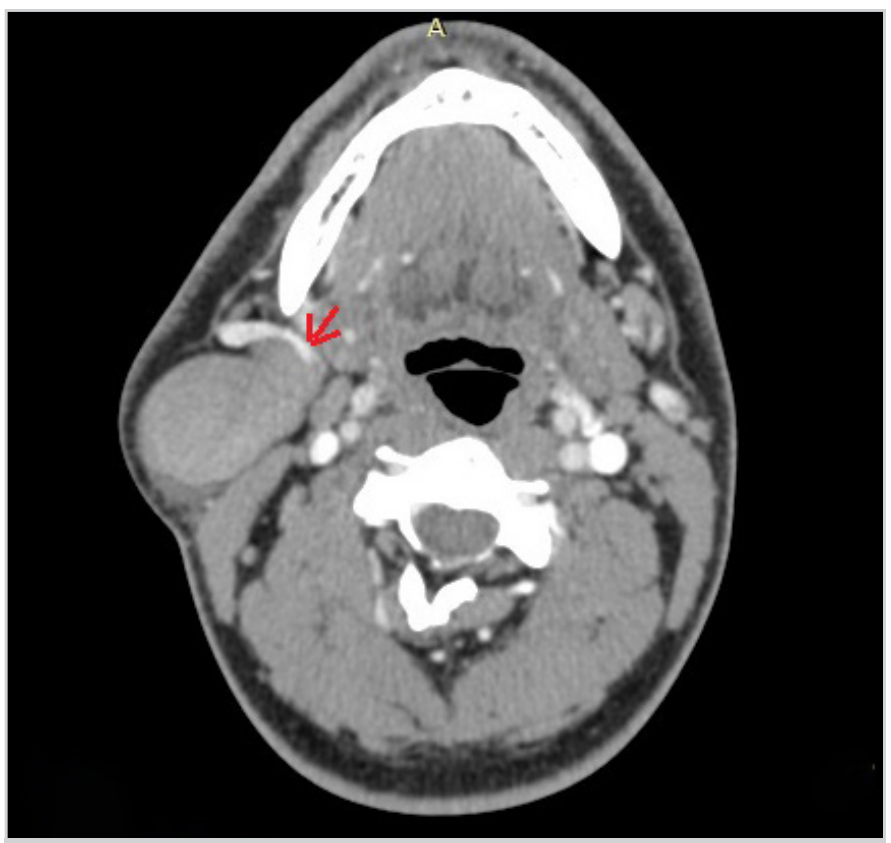

Figure 2. Axial images of the contrast enhanced computed tomography of the neck, showing venous ectasia of retromandibular vein (red arrow)

\section{Main Points}

- Venous ectasias or aneurysms are focal dilatations of the veins that are rare in the cervical region and usually misdiagnosed or ignored.

- They should be considered in the differential diagnosis of soft neck masses.

- Imaging has a key role in diagnosis.

- Conservative and surgical management have been attempted.

- Given the risk of thrombosis, thromboembolic events, rupture, and for aesthetic reasons, surgical excision is a sensible alternative.

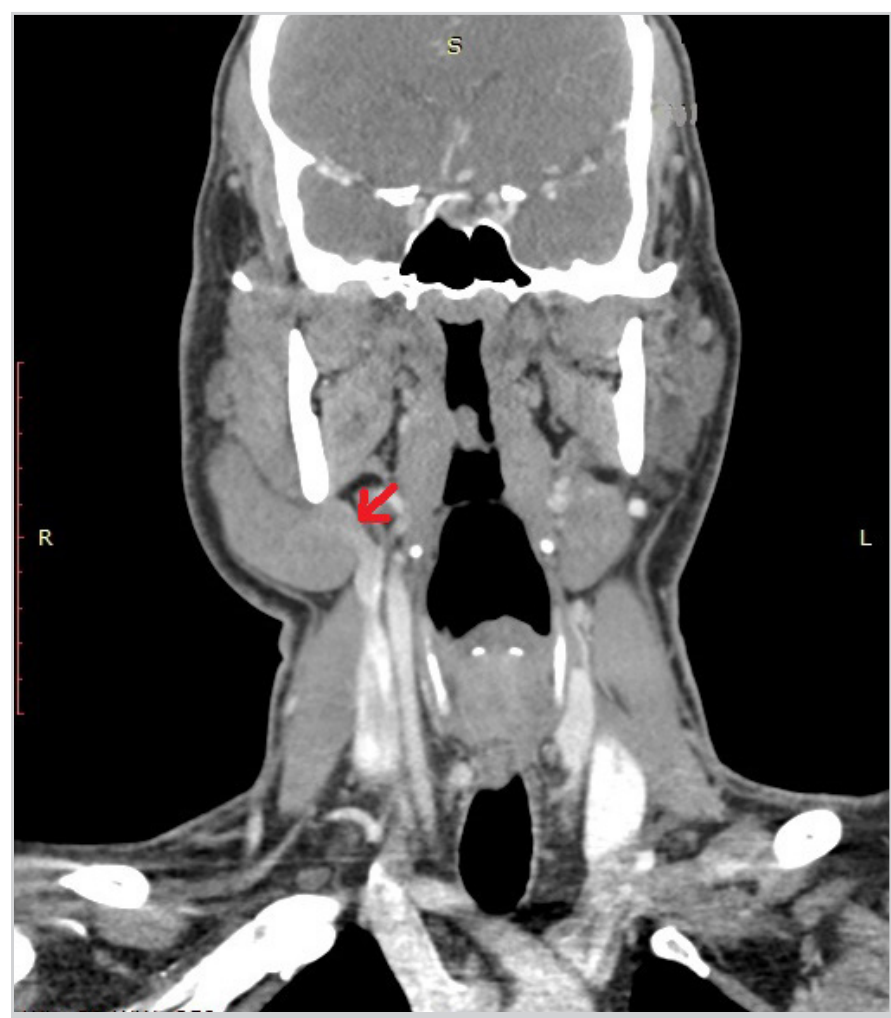

Figure 3. Coronal image of the lesion showing communication with common facial vein (red arrow)

patient; however, surgery was opted for because the lesion was directly draining into the major vein with high flow.

Excision of venous ectasia via transcervical approach was done under general anesthesia, after taking informed consent. A mass of ectatic venous channels $7 \times 4 \mathrm{~cm}$ was seen at the confluence of the facial vein, anterior division of retromandibular vein and common facial vein. We ligated the common facial vein. The ectatic venous channels were adherent with the external carotid artery, which was delineated and dissected out after ligating the anterior division of retromandibular vein, the facial vein proximally and the common facial vein distally as seen in Figure 4. The excised specimen is shown in Figure 5. The patient has been on regular follow-up for two months. Informed written consent was obtained from the patient for publication.

\section{Discussion}

Venous ectasias of the retromandibular vein and the facial vein are very rare lesions. To the best of our knowledge, this is the second case report in the literature but the exact incidence could not be given due to the rarity of the lesion (2). Venous aneurysms or ectasias with focal dilatation of the vein are uncommon. The first characterization was defined in 1915, after autopsy (3). The first described venous aneurysms were in 1928 (4). Venous ectasias in the neck usually have a benign course and generally are asymptomatic. They typically present with intermittent neck swelling that may aggravate following a Valsalva maneuver. 


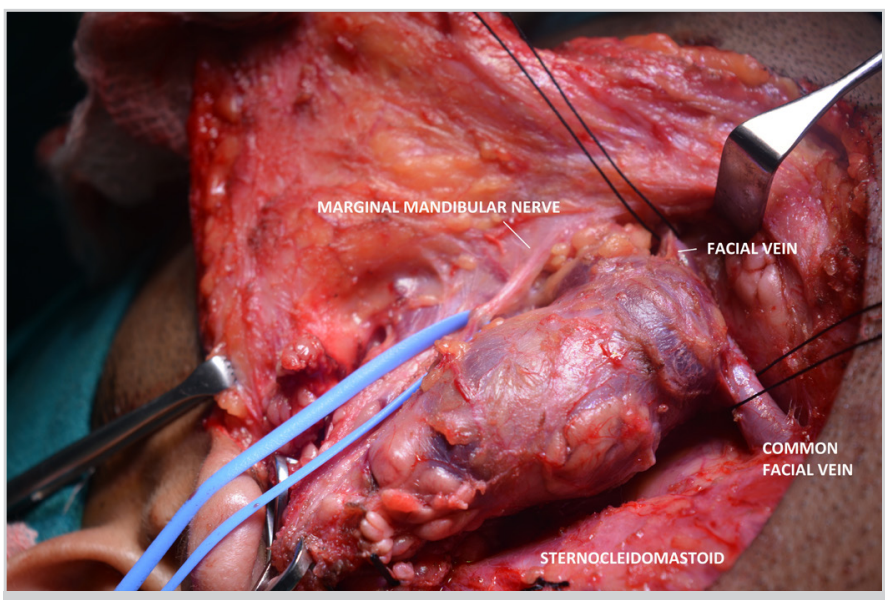

Figure 4. Intraoperative picture showing marginal mandibular nerve, facial vein and common facial vein along with lesion

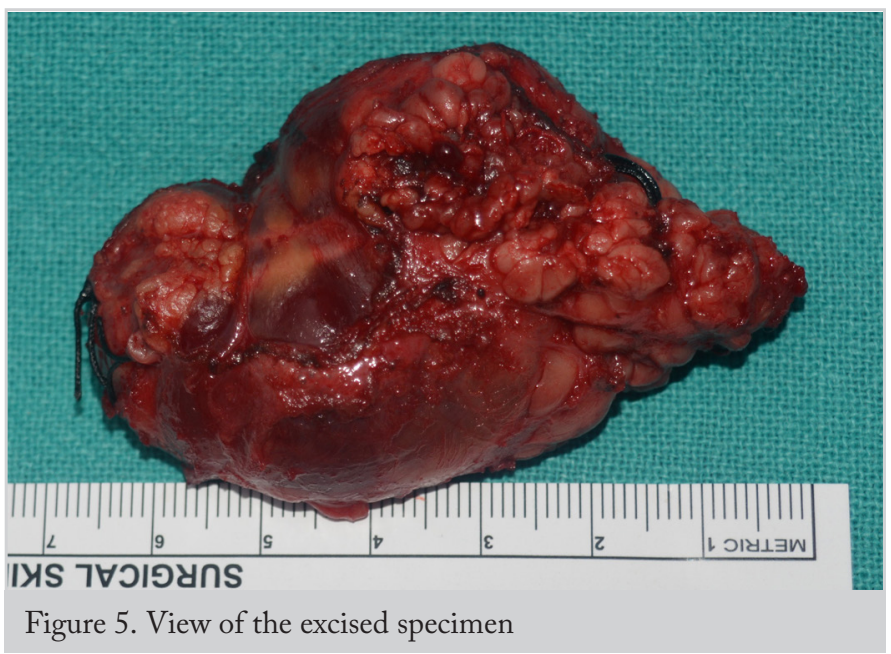

Several proposed mechanisms include infection, inflammation, mechanical trauma and venous hypertension (4). Some suggested that loss of focal connective tissue components of the vein wall would be possible due to congenital underdevelopment or degenerative connective tissue loss with age (5).

Differential diagnosis includes lymphangioma, branchial cyst, cystic hygroma, cavernous hemangioma, laryngocele, pharyngeal pouch, lymph node, thyroid mass or thyroglossal duct cyst. We should do a laryngeal examination to rule out laryngocele or pharyngeal pouch. Imaging is the key to diagnosis. Doppler ultrasonography is a readily reproducible imaging technique and has an advantage of accuracy (4). Contrast-enhanced computed tomography with angiography has equal diagnostic capability for lesion evaluation, as in our case. Its main advantage is that it allows the adjacent deeper structures of the neck to be better evaluated and provides a road map for the surgeon to plan the surgery (6). In our case, magnetic resonance imaging (MRI) was done to confirm the diagnosis and rule out other possible causes. MRI provides the benefit of evaluating the nonvascular structures in the neck that may not be well visualized with ultrasonography, without exposure to radiation (7).
Management generally depends on symptoms. Both conservative and surgical management have been attempted according to the literature. In jugular venous aneurysm, there is a high chance of thrombus formation within the neck veins due to low pressure and stagnant flow. Thrombus can lead to other consequences like pulmonary thromboembolism and stroke. Patients may present with pain over swelling in such a scenario. There is a risk of rupture of venous ectasia by trauma. Cosmetics was also a concern in our patient, therefore we preferred surgical excision. Surgical removal should be considered in the management of neck for the fear of thrombosis and possible rupture risks and cosmetic/aesthetic reasons.

Endovascular embolization of aneurysm was also done in some cases (8). But embolization may lead to residual swelling over the neck. This will necessitate excision. Post-embolization lesions have poor planes. This can lead to more intraoperative complications like nerve palsies.

Venous ectasias or venous malformations should also be suspected when a patient presents with intermittent neck swelling.

\section{Conclusion}

Venous ectasia of retromandibular and common facial veins is a rare vascular pathology. Doppler ultrasonography and CT angiography are very useful in the diagnosis. Surgery can be preferred instead of embolization in cases with potential risk of complications associated with particle migration due to rapid flow of the vascular pathology.

Informed Consent: Informed consent was obtained from the patient.

Peer-review: Externally peer-reviewed.

Author Contributions: Concept - D.K.; Supervision - A.G., V.S.; Data Collection and/or Processing - D.K., N.P.N.; Literature Search A.G.; Writing - N.P.N., V.M.; Critical Reviews - V.S., V.M.

Conflict of Interest: The authors have no conflicts of interest to declare.

Financial Disclosure: The authors declared that this study has received no financial support.

\section{References}

1. Mehta R, Kamble P, Nagarkar NM. Varied presentation of venous ectasia head and neck: case series and review of literature. Indian J Otolaryngol Head Neck Surg 2019; 71: 647-51.

2. Tanrıvermiş Sayit A, Çelenk Ç. Retromandibular venous ectasia mimicking a parotid mass: a rare case report with computerized tomography imaging findings. İstanbul Med J 2020; 21: 228-9.

3. Gabrielli R, Rosati MS, Siani A, Irace L. Management of symptomatic venous aneurysm. ScientificWorldJournal 2012; 2012: 386478. 
4. Verma RK, Kaushal D, Panda NK. External jugular vein aneurysm with thrombus presenting as painful neck mass: a case report. Oman Med J 2013; 28: 278-80.

5. Schatz IJ, Fine G. Venous aneurysms. N Engl J Med 1962; 266: 1310-2.

6. Desai SJ, Rajan S, Jain T. Anterior jugular vein aneurysm: diagnosis by CT angiography. Indian J Radiol Imaging 2002; 12: 369-70.
7. Bosshardt TL, Honig MP. Congenital internal jugular venous aneurysm: diagnosis and treatment. Mil Med 1996; 161: 2467.

8. Pandey NN, Sinha M, Deshpande A, Kumar S. External jugular vein aneurysm: successful endovascular management of an exceedingly rare entity. BMJ Case Rep 2020; 13: e233572 